\title{
The synthesis of poly(thiophene-co-fluorene) gradient copolymers
}

Tine Hardeman, Guy Koeckelberghs*

Laboratory for Polymer Synthesis, Division of Polymer Chemistry \& Materials, KU Leuven, Celestijnenlaan 200F, 3001 Heverlee (Leuven), Belgium

The copolymerization of thiophene and fluorene starting from a mixture of both monomers is investigated. It is shown that these monomers are incapable of copolymerizing using a Kumada catalyst transfer polycondensation. However, when the $\mathrm{Pd}(\mathrm{RuPhos})$-protocol is used, this copolymerization is enabled and gradient copolymers are obtained. This protocol is applied to synthesize a series of 6 polymers with varying monomer feed and the properties of these gradient copolymers are investigated. This new class of materials, which was previously inaccessible using other catalysts, shows unique properties compared to the block copolymer analogues.

\section{INTRODUCTION}

Conjugated copolymers containing fluorene are a very interesting class of materials for organic light-emitting diodes and photovoltaic applications. ${ }^{1}$ Up to now, these polymers were often 
synthesized via step-growth polymerization, which lacks control over molar mass, dispersity and end-groups. ${ }^{2-7}$ When Yokozawa ${ }^{8}$ and McCullough $^{9}$ independently discovered the controlled chain-growth nature of the Ni-catalyzed polymerization of 5-chloromagnesio-2-bromo-3hexylthiophene, it cleared the path for the synthesis of a variety of conjugated polymers with enhanced control. Not only poly(thiophene $)^{10-14}$, but also poly(phenylene) $)^{15,16}$, poly(pyrrole) ${ }^{17,18}$, poly(fluorene $)^{19-21}$, poly(selenophene $)^{22,23}, \quad$ poly(cyclopentadithiophene $)^{24}$ and poly(benzotriazole $)^{25,26}$ were synthesized using such type of controlled polymerization, as well as block copolymers of the aforementioned. ${ }^{2,3,20,27-30}$ The association of the catalyst to the polymer chain, allowing it to "walk" over the chain, was found to be at the origin of the controlled nature of these polymerizations. ${ }^{14,31}$ Because of this association, the catalyst does not diffuse away after the reductive elimination. It only participates in an intramolecular oxidative addition, hence converting this polycondensation into a chain-growth process. This concept of a 'catalyst transfer polymerization' (CTP) found many applications in the polymerization of various monomer systems, and resulted in much more convenient synthetic pathways towards block copolymers. However, problems arise when block copolymers with a specific order of addition or random copolymers are desired. Each monomer has different electronic properties and, as a consequence, a different affinity towards the catalyst. Because association is key in CTP, this difference in affinity can have detrimental effects on the polymerization. When the catalyst is associated to an electron-rich monomer, the transfer towards a monomer with less $\pi$-donor ability is difficult. This is the reason why block copolymerizations can only be successful when the more electron-rich monomer is added last (eg. poly(phenylene)- $b$-poly(thiophene) instead of poly(thiophene)- $b$ poly(phenylene) $)^{30,32}$, and it often complicates the synthesis of random copolymers via simply mixing both monomers and adding the catalyst. As a consequence, most random copolymers that 
are synthesized in a controlled way, consist of two monomer with similar properties, such as thiophenes with different side-chains. ${ }^{31,33-35}$ Another option is the synthesis of biaryl or even larger monomers. ${ }^{36-39}$ However, this generally results in a limited amount of possible copolymers, and requires lengthy monomer synthesis. An alternative solution can be found by using another pathway towards a controlled polymer synthesis. Instead of relying on the association of the catalyst, the $\mathrm{Pd}(\mathrm{RuPhos})$-protocol depends on deactivation of the monomers. ${ }^{40,41}$ In this protocol, bromo-bromozincio monomers are used, in combination with a Pd catalyst. The electron-donating organozinc group on the monomer deactivates the $\mathrm{C}-\mathrm{Br}$ bond for oxidative addition with $\operatorname{Pd}($ Ruphos). Hence, the monomers in solution are unable to react with the catalyst. The polymerization only starts when an initiator is added. This is typically a monomer without deactivating organozinc moiety, which can therefore undergo oxidative insertion with the Pd. Then the catalytic cycle with transmetalation, reductive elimination and oxidative insertion takes place. The oxidative insertion only occurs at the end of a growing polymer chain, because the deactivating organozinc moiety has reacted once a monomer is built in. In this type of polymerization, association of the catalyst to the polymer chain is not important.. Although it was found that some association generally takes place, the catalyst also diffuses away from the chain. However, this is not detrimental for the polymerization because the catalyst is stable in solution, and no sidereactions can occur because all monomers are deactivated. It is clear that this type of polymerization is much more suited to obtain random copolymers of two monomers with differing electronic properties.

Fluorene has shown much potential thanks to its highly fluorescent properties, and combinations with thiophene showed that energy transfer could occur between both. ${ }^{42}$ However, these polymers are all either synthesized via step-growth polymerization ${ }^{2-7}$, or they are synthesized using CTP, 
but then only the synthesis of block copolymers ${ }^{27,28}$ or alternating copolymers using biaryl monomers ${ }^{36}$ is investigated. We hypothesize that the differing affinity of thiophene and fluorene with respect to the Ni-catalyst does not allow for other variations, since growth of the polymer is likely to be discontinued when fluorene is built in after a series of thiophene monomer. As mentioned, the $\mathrm{Pd}(\mathrm{RuPhos})$-protocol is expected to eliminate these issues, as it is not dependent on catalyst association. As a consequence, it is independent of the nature of the monomers and should be able to copolymerize thiophene and fluorene monomers. This hypothesis is further supported by the fact that Verswyvel et al. have been able to synthesize block copolymers of these monomers in both direction, i.e. not only poly(fluorene)-b-poly(thiophene), but also poly(thiophene)- $b$-poly(fluorene).$^{40}$ The possibility to synthesize other copolymers of these two monomers is interesting, since research has shown that random or gradient copolymers tend to display a different set of properties compared to their block copolymer analogues. Especially gradient copolymers show unique solid state properties and phase behavior, which can be interesting for applications such as solar cells, transistors or light emitting diodes. Furthermore, they show good phase-compatibilizing properties when added to a mixture of the homopolymers. ${ }^{43-45}$ However, the number of gradient copolymers synthesized via a controlled polymerization is limited, for exactly the same reason as described above for random copolymers. Using CTP, only very similar monomers can be copolymerized, because otherwise difficulties arise with the cross-propagation. As a consequence, only thiophene/thiophene ${ }^{44,45}$ and thiophene/selenophene ${ }^{43}$ gradient copolymers have been synthesized so far. Because of the great similarity between the monomers, their reactivity was also found to be very similar and gradient copolymers could only be achieved via syringe pump addition of one of the monomers during the polymerization. 


\section{RESULTS AND DISCUSSION}

Random copolymer synthesis via CTP. First, the 'classic' CTP mechanism was used to investigate the effect of the differing affinity of the catalyst towards both monomers on the copolymerization of thiophene and fluorene. To this end, 2-bromo-5-chloromagnesio-3alkylthiophene and 2-bromo-7-chloromagnesio-9-dialkylfluorene were combined in a 50/50 ratio and added to the catalyst, $\mathrm{Ni}(\mathrm{dppp}) \mathrm{Cl}_{2}$ (Scheme 1$)$.

0.5

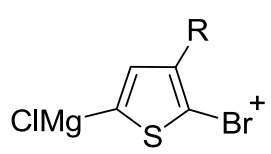
$R=$, N
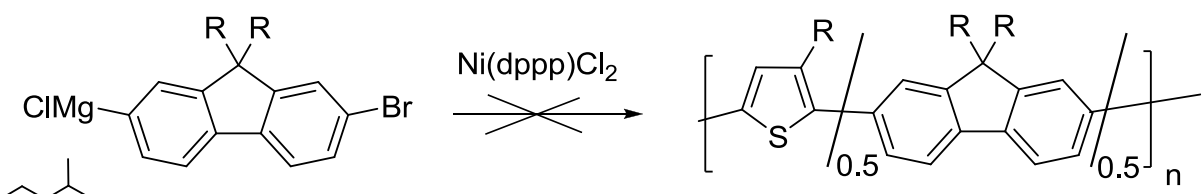

Scheme 1. Random copolymerization of thiophene and fluorene monomers via the CTP mechanism using $\mathrm{Ni}(\mathrm{dppp}) \mathrm{Cl}_{2}$.

After 1 hour, the polymer was precipitated in methanol. Although the precipitation clearly indicated that polymer was indeed formed, its purple color already suggested that mostly thiophene was incorporated in the polymer chain. Analysis of the filtrate indeed showed that almost exclusively fluorene monomer was left unreacted in a substantial amount. These observations were further confirmed by ${ }^{1} \mathrm{H}$ NMR analysis of the polymer (Figure S1). It is calculated that this polymer contains only $13 \%$ fluorene, despite the fact that $50 \%$ was present in the monomer feed. As a consequence, it is concluded that the formation of a random thiophene/fluorene copolymer from a mixture of both monomers is not possible using CTP. Consequently, the attention is shifted to the $\mathrm{Pd}(\mathrm{RuPhos})$-protocol.

Monomer synthesis. Precursor monomers 1 and $\mathbf{2}$ are synthesized equivalent to literature procedures. ${ }^{41,46}$ Both are equipped with a chiral side-chain, enabling a more thorough investigation of the aggregation behavior. To convert these precursor monomers to the actual monomers used 
in the polymerization, first a Grignard metathesis with either $t \mathrm{BuMgCl}$ (thiophene) or $t \mathrm{Bu} 3 \mathrm{MgLi}$ (fluorene) is used, after which transmetalation with dried $\mathrm{ZnBr}_{2}$ is performed (Scheme 2). It is essential to work in very dry conditions, since protonated monomer will act as a transfer agent in Pd(RuPhos) polymerizations.<smiles>[R]c1cc(I)sc1Br</smiles>

$\stackrel{1 \text { eq } t \mathrm{BuMgCl}}{\longrightarrow}$
$R$<smiles>[R]C1([R])c2cc(Br)ccc2-c2ccc(I)cc21</smiles><smiles>[R]c1cc(C)sc1Br</smiles><smiles>[13CH2][131In]</smiles><smiles>[R]C1=[Y](Br)=[S+]C(Br)=C1</smiles><smiles>[R]C1([R])c2cc(C)ccc2-c2ccc(Br)cc21</smiles>
$R=$, /

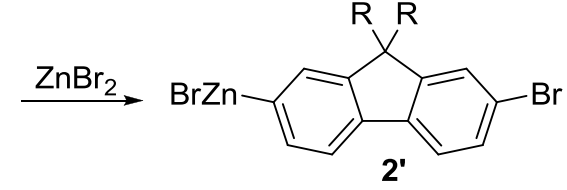

Scheme 2. Conversion of the precursor monomers 1 and $\mathbf{2}$ to the monomers used in the $\mathrm{Pd}(\mathrm{RuPhos})$ polymerization (1' and 2') via Grignard metathesis and transmetalation with $\mathrm{ZnBr}_{2}$.

Polymer synthesis. Once the monomers are prepared, they are combined in different ratios and added to the catalyst (Scheme 3). The molar mass and dispersity is shown for each polymer in Table 1 . Monomer conversions of around $75 \%$ are typically observed.

a<smiles>[R]CCC(C)CCC(C)CCCCC(=O)c1ccc2c(c1)C([R])([R])c1cc(Br)ccc1-2</smiles>
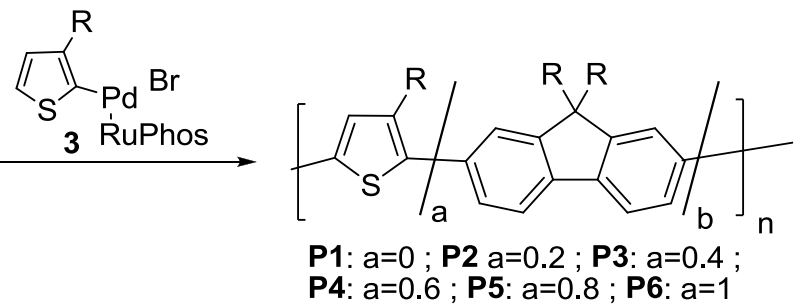

Scheme 3. Synthesis of the thiophene/fluorene copolymers via the $\mathrm{Pd}(\mathrm{RuPhos})$ protocol.

The ${ }^{1} \mathrm{H}$ NMR spectra of all polymers are shown in Figure 1. Making use of the $\alpha$-methylene protons, it is determined that - in contrast to the polymerization with $\mathrm{Ni}(\mathrm{dppp}) \mathrm{Cl}_{2}$ - the monomer feed used corresponds well to the composition found in the polymer (Table 1). Hence, it is shown 
that the polymerization of a thiophene/fluorene monomer mixture is enabled via the $\mathrm{Pd}(\mathrm{RuPhos})$ protocol, and that both monomers are indeed incorporated in the process, something which is not possible via catalyst transfer polymerizations.

Table 1. Aimed $\left(\boldsymbol{a}_{\boldsymbol{t h}}\right)$ and obtained $\left(\boldsymbol{a}_{\text {exp }}\right)$ monomer ratios, Number average molar mass $\left(\bar{M}_{\mathrm{n}}\right)$ and dispersity (Đ) of the copolymers.

\begin{tabular}{ccccc}
\hline Pol & $a_{t h}$ & $a_{\text {exp }}$ & $\begin{array}{c}\bar{M}_{\mathrm{n}} \\
(\mathrm{kg} / \mathrm{mol})\end{array}$ & Đ \\
\hline P1 & 0.00 & 0.00 & 8.9 & 1.5 \\
P2 & 0.20 & 0.24 & 5.2 & 1.6 \\
P3 & 0.40 & 0.48 & 4.2 & 1.5 \\
P4 & 0.60 & 0.61 & 4.6 & 1.5 \\
P5 & 0.80 & 0.76 & 5.3 & 1.5 \\
P6 & 1.00 & 1.00 & 6.1 & 1.5 \\
\hline
\end{tabular}




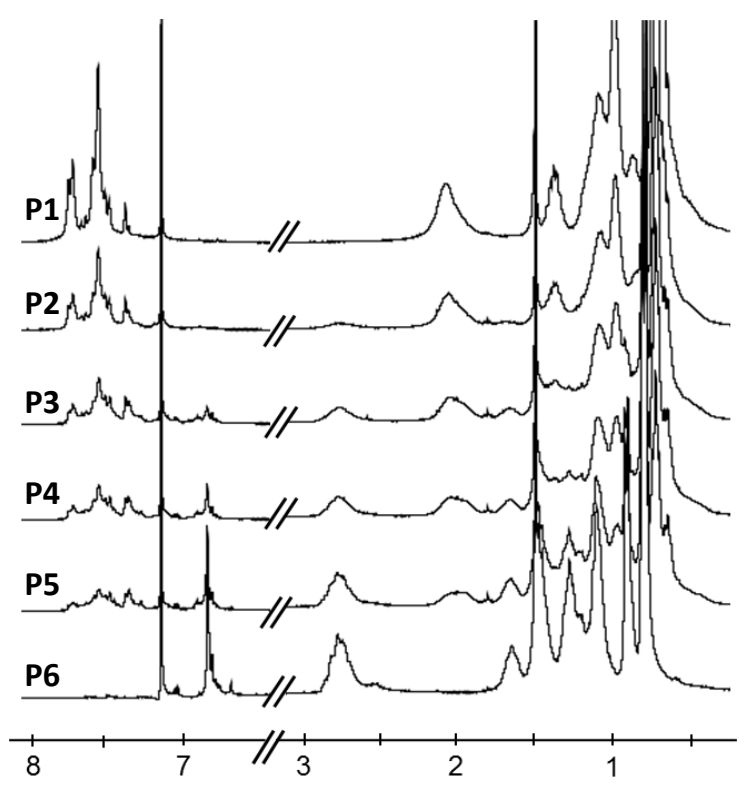

Figure 1. ${ }^{1} \mathrm{H}$ NMR spectrum of the thiophene/fluorene copolymers.

Lewis-Mayo analysis. Now that the monomer ratio is established in all polymers, the next question is whether the polymers are blocky, have a gradient or are fully random copolymers. This can be determined by making use of a Lewis-Mayo plot. In this plot, experimental data are fitted with the Lewis-Mayo equation, shown below for copolymerization of monomers A and B.

$$
F_{A}=1-F_{B}=\frac{r_{A} f_{A}^{2}+f_{A} f_{B}}{r_{A} f_{A}^{2}+2 f_{A} f_{B}+r_{B} f_{B}^{2}}
$$

In this equation, $f_{A}$ and $f_{B}$ represent the fractions of monomer $\mathrm{A}$ and $\mathrm{B}$ in the monomer feed, while $F_{A}$ and $F_{B}$ represent the fractions of $\mathrm{A}$ and $\mathrm{B}$ in the increment polymer, which corresponds to the composition at low conversion. This last part is essential, because only the situation at low conversion shows which monomer is being built in preferentially. $\mathrm{r}_{\mathrm{A}}$ and $\mathrm{r}_{\mathrm{B}}$, the values which are determined via the plot, are given by the following equations.

$$
r_{A}=\frac{k_{A A}}{k_{A B}}
$$




$$
r_{B}=\frac{k_{B B}}{k_{B A}}
$$

$\mathrm{k}_{\mathrm{AA}}$ is the rate constant for reaction of monomer $\mathrm{A}$ at the polymer chain end with another monomer $\mathrm{A}$, while $\mathrm{k}_{\mathrm{AB}}$ gives the rate constant of the same polymer chain end reacting with monomer $\mathrm{B}$. Hence, the ratio between both, $\mathrm{r}_{\mathrm{A}}$, shows which monomer is preferably incorporated when monomer A is located at the polymer chain end. Similarly, $\mathrm{r}_{\mathrm{B}}$ gives the ratio of the rate constants for reaction of a polymer chain with $\mathrm{B}$ at the chain end for reaction with another monomer $\mathrm{B}\left(\mathrm{k}_{\mathrm{BB}}\right)$ or monomer $\mathrm{A}\left(\mathrm{k}_{\mathrm{BA}}\right)$.

To be able to make the Lewis-Mayo plot, the polymerization is repeated but quenched at low conversion using acidified THF. Furthermore, an internal reference (1,2,3-trimethoxybenzene) is added to the monomer mixtures to be able to determine the depletion of both monomers via GPC analysis. By comparing the GPC spectrum of each monomer mixture with the one from the quenched polymerization, the fractions of monomer incorporated into the polymer at low conversion can be determined. This resulted in the Lewis-Mayo plot shown in Figure 2. 


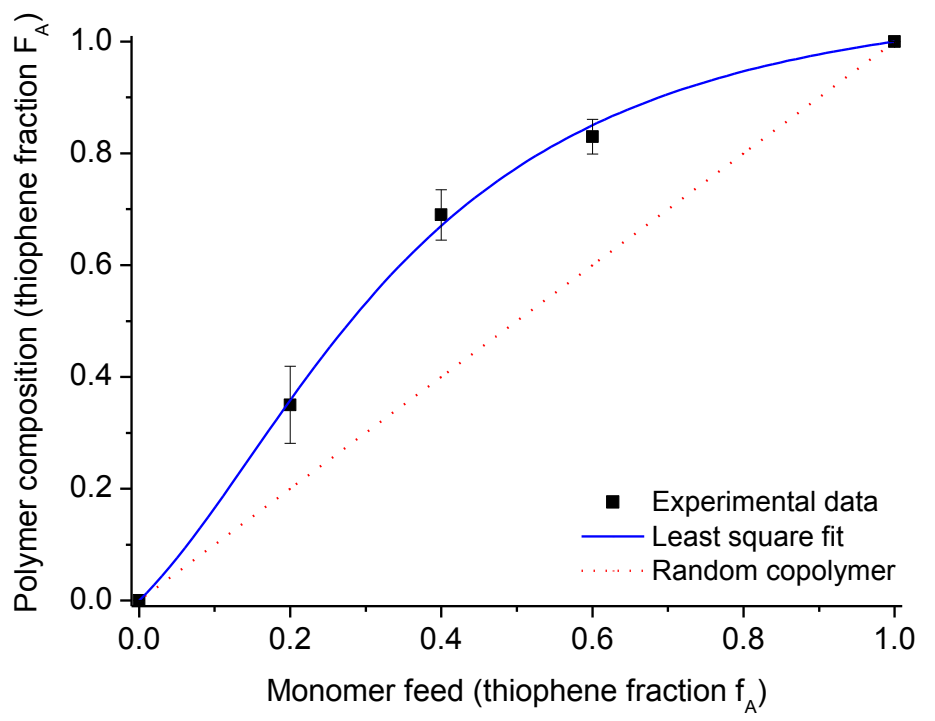

Figure 2. Lewis-Mayo plot for the thiophene/fluorene copolymerization. The data points obtained for $\mathrm{f}_{\mathrm{A}}=0.8$ are not included, since they were repeatedly obtained at too high conversion $(\sim 30 \%)$.

Since it was found that the polymerization rate was very high, the polymerizations were all quenched within 10 s after all monomer was added. However, for P5 (80\% thiophene monomer) the polymerization rates were found to be so high that quenching at conversions lower that $25 \%$ was not possible. Even after immediately adding acidified THF after transfer of the monomer, sufficiently low conversions could not be obtained. Therefore, the datapoint is not included to determine $r_{A}$ and $r_{B}$. The least square fit gave following results for $r_{A}$ (thiophene) and $r_{B}$ (fluorene):

$$
\begin{aligned}
& r_{A}=5.0 \pm 0.9 \\
& r_{B}=0.8 \pm 0.1
\end{aligned}
$$

This means that when a thiophene is present at the end of a polymer chain, reaction with another thiophene monomer is 5 times faster than reaction with a fluorene monomer. If we assume, for example, that fluorene and thiophene are present in equal amounts, this corresponds to a $83 \%$ chance of thiophene becoming the next repeating unit. When a fluorene monomer is at the end of 
a chain, again it is slightly more likely for a thiophene to be the next monomer (55\%). This data could be linked to the fact that Verswyvel et al. also found the polymerization of thiophene to proceed much faster than the fluorene polymerization using the $\mathrm{Pd}(\mathrm{RuPhos})$ protocol. As a consequence, mostly thiophene is incorporated in the beginning of the polymer chain. Fluorene is built in at the end of the polymerization upon depletion of the thiophene monomer, resulting in a gradient type of copolymer. Because there is a large difference in $r_{A}$ and $r_{B}$, the composition of the polymer is highly dependent on the conversion. This further supports our choice to not include P5 to calculate the Lewis-Mayo least square fit, since the conversion was considerably higher for that sample.

To overcome the problem of the very fast polymerization for $\mathbf{P 5}$, inhibiting the acquisition of that data point for the Lewis-Mayo plot, the entire experiment was repeated at $0^{\circ} \mathrm{C}$. Because of the lower temperature, all polymerizations slowed down considerably and data point could be obtained for all monomer feeds (Figure S4). At $0^{\circ} \mathrm{C}, \mathrm{r}_{\mathrm{A}}$ equals $4.6 \pm 1.0$ and $\mathrm{r}_{\mathrm{B}}$ equals $0.95 \pm 0.2$. These values are slightly lower than the ones obtained at $25^{\circ} \mathrm{C}$, although the differences fall within the error margins.

Predicted molecular structure. To verify how the difference in reaction rates affects the polymers with varying monomer feeds, the instantaneous polymer composition with conversion is calculated using the equations developed by $\mathrm{Skeist}^{47}$ and further elaborated by Mayo and Walling. ${ }^{48}$ These calculations predict that the polymer composition varies substantially with conversion, and that the effect thereof on the resulting molecular structure also largely depends on the initial monomer feed (Figure 3). 


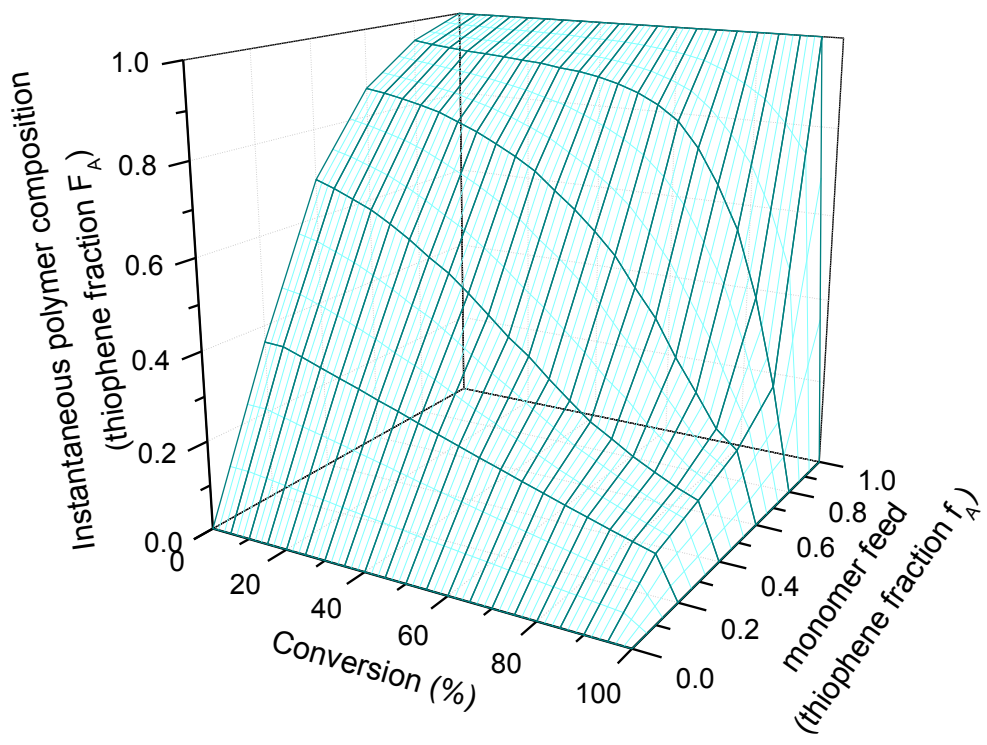

Figure 3. Instantaneous polymer composition (thiophene fraction $\mathrm{F}_{\mathrm{A}}$ ) versus conversion and monomer feed (thiophene fraction $\mathrm{f}_{\mathrm{A}}$ ).

First of all, these calculations again illustrate the general trend that thiophene monomers incorporated more than proportional at low conversion. Graphically this can be concluded by all data points lying above the diagonal in the plane at $0 \%$ conversion. As the polymerization proceeds and depletion of the thiophene monomer occurs, more and more fluorene is built in, resulting in polymer chain ends consisting largely of fluorene sequences. This is demonstrated by all data points lying below the diagonal in the plane at $100 \%$ conversion.

To get an idea of the molecular structure for each polymer (P1 to P5), the instantaneous polymer composition should be evaluated going from 0 to $100 \%$ conversion at the respective monomer feed. (Note that this is only true for controlled chain-growth polymerizations, where all chains are initiated simultaneously at the beginning and then grow continuously during the polymerization.) For the polymer with a large excess of thiophene monomer in the feed $\left(\mathbf{P 5}, \mathrm{f}_{\mathrm{A}}=0.8\right)$, a clear transition can be observed. Almost exclusively thiophene monomer is built in up to around $70 \%$ 
conversion ( $\mathrm{F}_{\mathrm{A}}$ slowly decreases from 0.95 at $0 \%$ conversion to 0.82 at $70 \%$ conversion), after which fluorene starts to be incorporated substantially as well (steep drop in $\mathrm{F}_{\mathrm{A}}$ to 0.24 ). As a consequence, a quite blocky structure is expected for this polymer, but with a relatively short fluorene block because the latter is present in much smaller amounts. A similar trend is observed for $\mathbf{P 4}$ and $\mathbf{P 3}$, but it is systematically less pronounced. As a consequence, the blockiness diminishes and a gradient structure is expected for these copolymers. When a large amount of fluorene is present in the monomer feed $\left(\mathbf{P 2}, \mathrm{f}_{\mathrm{A}}=0.2\right)$, depletion of thiophene occurs quite fast and almost exclusively fluorene is built in at higher conversions. Hence, P2 is again expected to show a more blocky structure, but now with a large fluorene block. To conclude, while gradient copolymers are always obtained, we expect that both P2 and P5 show a more blocky structure compared to the others (P3 and P4).

${ }^{1}$ H NMR analysis. To verify the molecular structure of the polymers, the ${ }^{1} \mathrm{H}$ NMR spectrum is again considered, with a focus on the aromatic region. Although a determination of the randomness purely based on this NMR is not possible because of the abundance and overlap of peaks arising from the fluorene monomer, it is possible to get some qualitative information (Figure 4). The homopolymers provide spectra showing only thiophene-thiophene or fluorene-fluorene couplings, enabling an easy assignment of these peaks in other spectra. If a block copolymer is formed, the spectrum resembles a summation of the spectra of the respective homopolymers, taking into account the relative abundance of both and keeping in mind that some extra peaks would arise from the link between both blocks. If, on the other hand, a random copolymer is formed, the large amount of thiophene-fluorene and fluorene-thiophene cross couplings results in a much larger variety of peaks in the aromatic region. Applying this to the set of copolymers, it is observed that 
$\mathbf{P 2}$ and $\mathbf{P 5}$ indeed largely resemble the homopolymers (P1 and $\mathbf{P 6}$, respectively), indicating that a fluorene block for the first, and a thiophene block for the latter is present. While the signal of the fluorenes is also clearly visible for $\mathbf{P 5}$, the peak at $6.97 \mathrm{ppm}$ assigned to thiophene-thiophene couplings is not present for $\mathbf{P 2}$. Hence, an actual thiophene block is not present for this polymer, it is simply incorporated along with the fluorene at low conversion until fluorene takes over upon depletion of the thiophene monomer. Similarly, also the 'fluorene block' in P5 is expected to still contain thiophene monomer. The intermediate situation (peaks arising from both homocouplings and cross couplings) observed for $\mathbf{P} \mathbf{3}$ and $\mathbf{P 4}$ suggests the formation of gradient copolymers

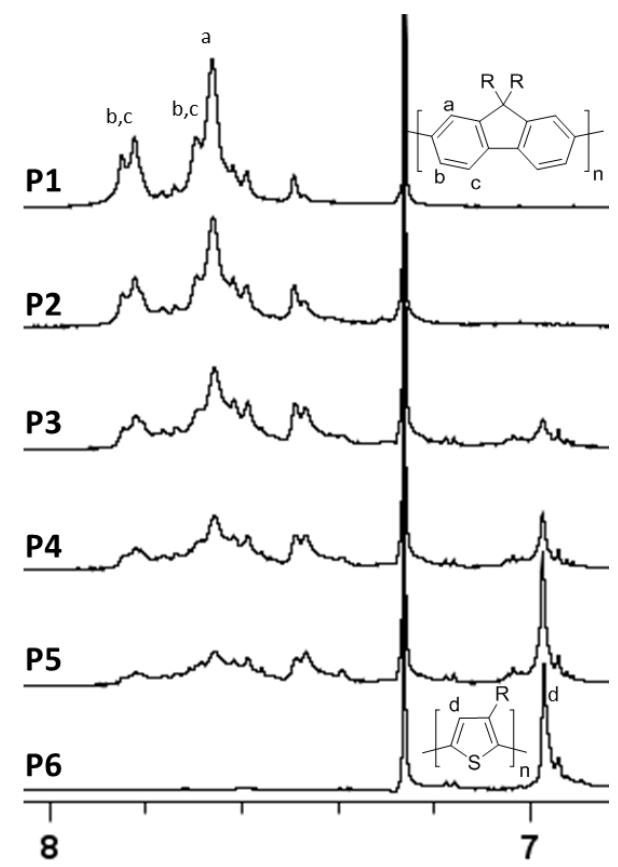

Figure 4. Aromatic region of the ${ }^{1} \mathrm{H}$ NMR spectra of the polymers.

Optical properties. The picture painted by the theoretical calculations and ${ }^{1} \mathrm{H}$ NMR analysis is confirmed by the optical properties observed for these polymers. Comparison of the absorption spectra of these polymers in $\mathrm{CHCl}_{3}$ solution (Figure 5) with the ones for poly(fluorene)-bpoly(thiophene) $)^{27}$ and alternating copolymers (but with varying amounts of thiophene in the repeating unit) ${ }^{3}$, clearly demonstrates more resemblance with the block copolymer. A shift in $\lambda_{\max }$, 
as would be expected for a random copolymer, is hardly observed and the fluorene and thiophene sequences appear to be absorbing light more or less independently from each other. Nevertheless, the bimodal absorption spectrum observed for the actual block copolymer is not that pronounced for most copolymers, again evidencing the formation of gradient copolymers. The only exception is P5, which does show a clear bimodal absorption spectrum. This is in agreement with the expectation that this polymer would have the most blocky structure.

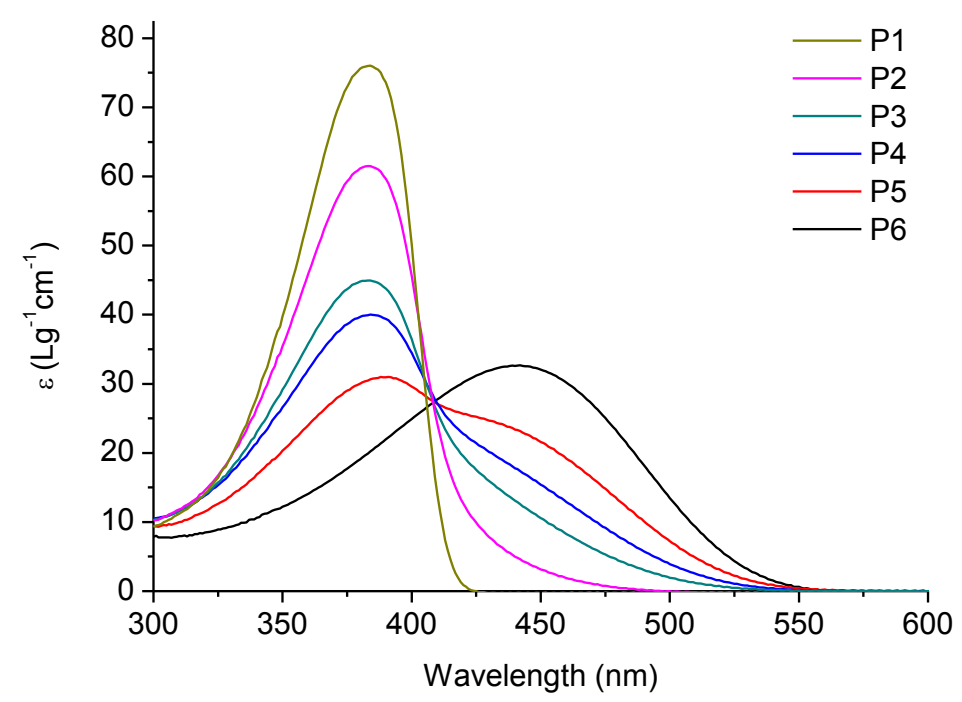

Figure 5. Absorption spectra of the thiophene/fluorene copolymers in $\mathrm{CHCl}_{3}(\mathrm{c}=40 \mathrm{mg} / \mathrm{mL})$.

The fluorescence of the polymers, shown in Figure 6, also shows independent fluorescence of thiophene and fluorene sequences. Again, this is most pronounced for P5. For the other copolymers, slightly enhanced fluorescence in the region around 500-520 nm is observed, which might be attributed to the fluorescence of thiophene-fluorene mixed species present in the gradient copolymer. A clear enhancement of the fluorescence of polythiophene in the copolymer with respect to the homopolymer is not observed, indicating that Förster transfer does not occur efficiently in these polymers. 


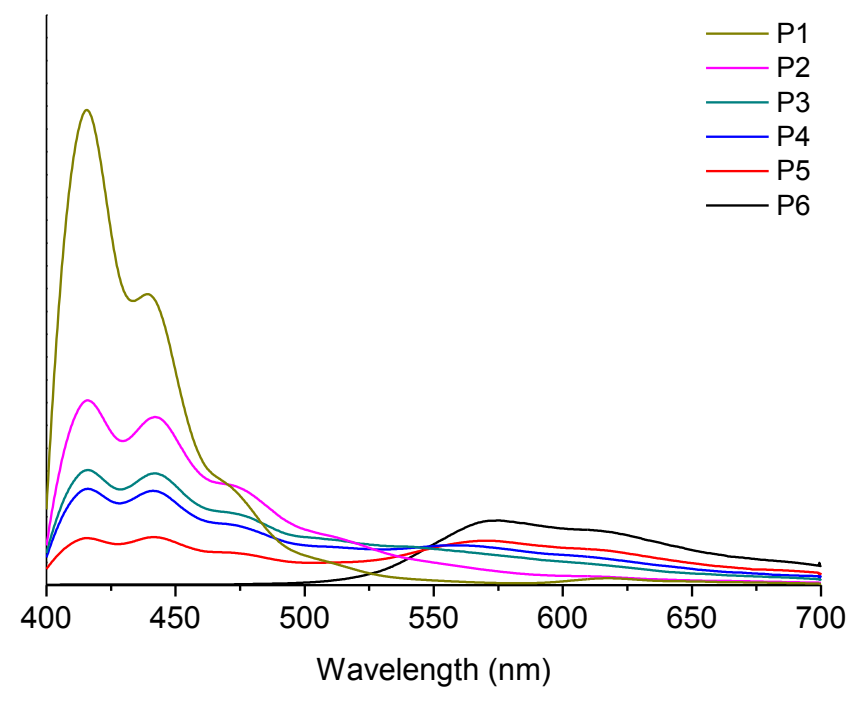

Figure 6. Fluorescence of the thiophene/fluorene copolymers in $\mathrm{CHCl}_{3}(\mathrm{c}=1.2 \mathrm{mg} / \mathrm{L})$. The intensities are corrected for the different absorption at the excitation wavelength $\left(\boldsymbol{\lambda}_{\boldsymbol{e x}}=385 \mathrm{~nm}\right)$.

Chiral self-assembly. The aggregation behavior of the polymers also reveals information on the structure of the materials. Stacking is in general significantly complicated upon formation of a random copolymer structure. ${ }^{38}$ As expected, homopolymers P1 and P6 show efficient stacking upon addition of methanol to the polymer solution in chloroform. This is evidenced by the shift in $\lambda_{\max }$ in the UV-Vis absorption spectrum and the appearance of a large CD signal, which indicates the formation of chiral aggregates (Figure 7 and Figure S6). The most significant CD signal in the copolymers is found for P5, which clearly shows chiral stacking in the thiophene absorption region. Furthermore, it also shows a shoulder around $620 \mathrm{~nm}$ in the UV-Vis absorption spectrum, known to originate from long range order in the aggregates. This is again in agreement with the formation of a more blocky copolymer, of which the thiophene block starts to aggregate upon addition of methanol. P4 also shows some CD signal in this region, but only to a very small extent, indicating that an actual thiophene block is probably not present. P3 does not seem to stack 
properly in bad solvent conditions, but for $\mathbf{P} 2$ again some CD signal can be seen in the fluorene absorption region from 350 to $450 \mathrm{~nm}$. Although the signal is relatively small, this does indicate that after depletion of the thiophene monomer (only present for $20 \%$ in the monomer feed), a fluorene block capable of stacking, is formed.
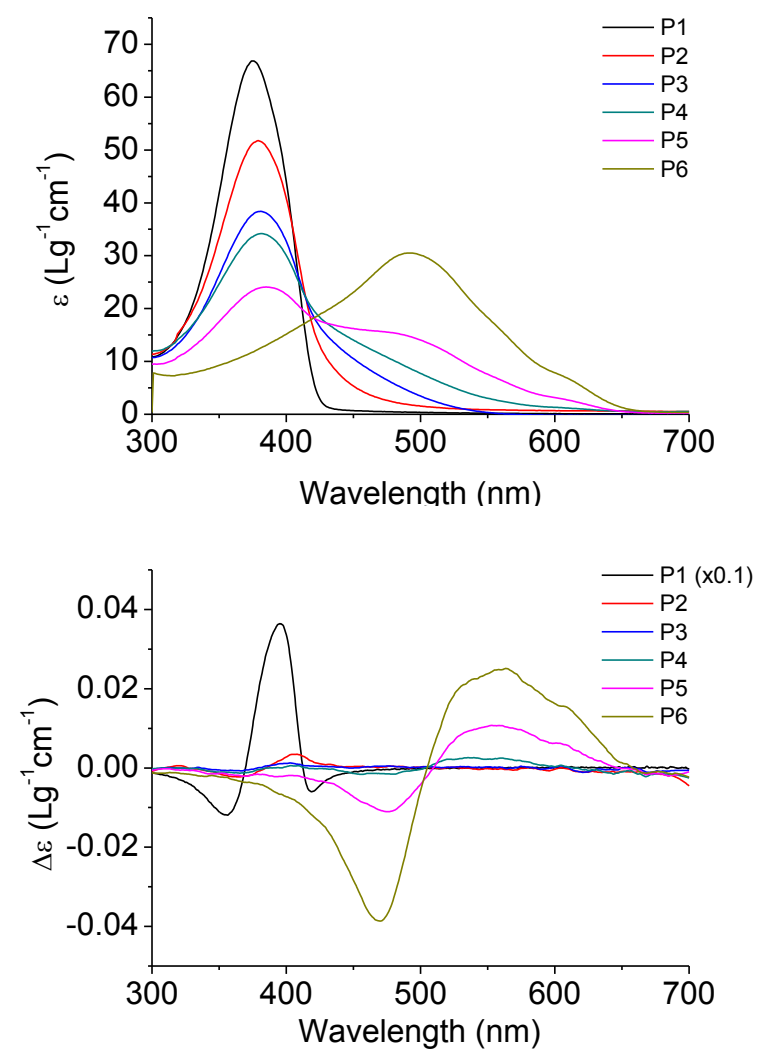

Figure 7. Absorption (above) and CD (below) spectra of all thiophene/fluorene copolymers in a 90/10 Chloroform/Methanol solvent mixture. Spectra recorded for different solvent mixtures are given in the ESI (Figure S6).

Solid state properties. Finally, melting and crystallization behavior of the polymers is investigated using DSC (Figure S7). Only for P6, regular poly(thiophene), clear melting and crystallization peaks are observed at $109^{\circ} \mathrm{C}$ and $44^{\circ} \mathrm{C}$ respectively. For $\mathbf{P 5}$, some small signal is 
observed during heating, but it is ill defined. These observations were to be expected, as both polymers showed a shoulder at $620 \mathrm{~nm}$ in the absorption spectrum at high methanol content.

\section{CONCLUSION}

The copolymerization of thiophene and fluorene monomer mixtures in a controlled polymerization was attempted via a catalyst transfer polycondensation using $\mathrm{Ni}(\mathrm{dppp}) \mathrm{Cl}_{2}$. However, it was found that fluorene was hardly incorporated and poly(thiophene)-like polymers were obtained. This was ascribed to the fact that catalyst association is key in these types of polymerizations, and the reduced affinity of the catalyst towards fluorene with respect to thiophene is detrimental for the polymerization. On the other hand, the different association between the monomers and the catalyst is of no importance in the $\mathrm{Pd}(\mathrm{RuPhos})$ protocol, making it very flexible. When thiophene/fluorene monomer mixtures are copolymerized using this mechanism, the polymer composition corresponds well to the ratio used in the monomer feed. However, it was found that the polymerization of thiophene occurs much faster than the fluorene polymerization, resulting in gradient copolymers. In the beginning of the polymerization, thiophene is mostly built in and the largest part of the fluorene incorporation only occurs after depletion of the thiophene

monomer. For the copolymer with $80 \%$ thiophene monomer in the feed, this resulted in a significant thiophene block in the polymer chain. The other polymers are better described by having a gradient, although when only $20 \%$ of thiophene is present in the feed, almost exclusively fluorene can be found near the end of the polymer chains, resulting also in a fluorene block capable of stacking..

\section{ASSOCIATED CONTENT}


Supporting Information. Reagents and instrumentation, synthetic procedures for $\mathbf{1}$ and the monomers (1' and 2'), polymerization procedures, NMR spectrum of the copolymer synthesized via $\mathrm{KCTP}$, the effect of the soxhlet extraction with methanol, the results for a chain-extension experiment, method for obtaining Lewis-Mayo plot and the raw data, all absorption and CD spectra recorded for the polymers at different solvent conditions, DSC data. This material is available free of charge via the Internet at http://pubs.acs.org.

\section{AUTHOR INFORMATION}

\section{Corresponding Author}

*E-mail: Guy.Koeckelberghs@chem.kuleuven.be

\section{Author Contributions}

The manuscript was written through contributions of all authors. All authors have given approval to the final version of the manuscript

\section{Notes}

The authors declare no competing financial interest.

\section{ACKNOWLEDGMENT}

We are grateful to the Onderzoeksfonds K.U.Leuven/Research Fund K.U.Leuven and the Fund for Scientific Research (FWO-Vlaanderen) for financial support. T.H. is a doctoral fellow of the Fund for Scientific Research (FWO- Vlaanderen).

\section{REFERENCES}


(1) Grimsdale, A. C.; Chan, K. L.; Martin, R. E.; Jokisz, P. G.; Holmes, A. B. Chem. Rev. 2009, 109 (3), 897-1091.

(2) Scherf, U.; Gutacker, A.; Koenen, N. Acc. Chem. Res. 2008, 41 (9), 1086-1097.

(3) Liu, B.; Yu, W. L.; Lai, Y. H.; Huang, W. Macromolecules 2000, 33, 8945-8952.

(4) Inagi, S.; Hayashi, S.; Hosaka, K.; Fuchigami, T. Macromolecules 2009, 42 (12), 38813883 .

(5) Kreyenschmidt, M.; Klaerner, G.; Fuhrer, T.; Ashenhurst, J.; Karg, S.; Chen, W. D.; Lee, V. Y.; Scott, J. C.; Miller, R. D. Macromolecules 1998, 31, 1099-1103.

(6) Lee, J.-I.; Klaerner, G.; Davey, M. H.; Miller, R. D. Synth. Met. 1999, 102 (1-3), 10871088 .

(7) Paulsen, B. D.; Speros, J. C.; Claflin, M. S.; Hillmyer, M. a.; Frisbie, C. D. Polym. Chem. 2014, 5 (21), 6287-6294.

(8) Yokoyama, A.; Miyakoshi, R.; Yokozawa, T. Macromolecules 2004, 37, 1169-1171.

(9) Sheina, E. E.; Liu, J.; Iovu, M. C.; Laird, D. W.; McCullough, R. D. Macromolecules 2004, 37 (10), 3526-3528.

(10) Miyakoshi, R.; Yokoyama, A.; Yokozawa, T. J. Am. Chem. Soc. 2005, 127 (6), 1754217547.

(11) Yokozawa, T.; Suzuki, R.; Nojima, M.; Ohta, Y.; Yokoyama, A. Macromol. Rapid Commun. 2011, 32, 801-806.

(12) Lanni, E. L.; Mcneil, A. J. J. Am. Chem. Soc. 2009, 131 (13), 16573-16579.

(13) Beryozkina, T.; Senkovskyy, V.; Kaul, E.; Kiriy, A. Macromolecules 2008, 41 (21), 7817-7823.

(14) Tkachov, R.; Senkovskyy, V.; Komber, H.; Sommer, J. U.; Kiriy, A. J. Am. Chem. Soc. 2010, 132, 7803-7810.

(15) Miyakoshi, R.; Shimono, K.; Yokoyama, A.; Yokozawa, T. J. Am. Chem. Soc. 2006, 128, $16012-16013$.

(16) Yokozawa, T.; Kohno, H.; Ohta, Y.; Yokoyama, A. Macromolecules 2010, 43, 70957100.

(17) Stefan, M. C.; Javier, A. E.; Osaka, I.; McCullough, R. D. Macromolecules 2009, 42 (1), 30-32. 
(18) Yokoyama, A.; Kato, A.; Miyakoshi, R.; Yokozawa, T. Macromolecules 2008, 41, 72717273.

(19) Huang, L.; Wu, S.; Qu, Y.; Geng, Y.; Wang, F. Macromolecules 2008, 41, 8944-8947.

(20) Sui, A.; Shi, X.; Wu, S.; Tian, H.; Geng, Y.; Wang, F. Macromolecules 2012, 45 (13), $5436-5443$.

(21) Yokoyama, A.; Suzuki, H.; Kubota, Y.; Ohuchi, K.; Higashimura, H.; Yokozawa, T. J. Am. Chem. Soc. 2007, 129, 7236-7237.

(22) Heeney, M.; Zhang, W.; Crouch, D. J.; Chabinyc, M. L.; Gordeyev, S.; Hamilton, R.; Higgins, S. J.; McCulloch, I.; Skabara, P. J.; Sparrowe, D.; Tierney, S. Chem. Commun. (Camb). 2007, 5061-5063.

(23) Hollinger, J.; Jahnke, A. A.; Coombs, N.; Seferos, D. S. J. Am. Chem. Soc. 2010, 132 (25), 8546-8547.

(24) Willot, P.; Govaerts, S.; Koeckelberghs, G. Macromolecules 2013, 46, 8888-8895.

(25) Bridges, C. R.; McCormick, T. M.; Gibson, G. L.; Hollinger, J.; Seferos, D. S. J. Am. Chem. Soc. 2013, 135 (35), 13212-13219.

(26) Bridges, C. R.; Yan, H.; Pollit, A. A.; Seferos, D. S. ACS Macro Lett. 2014, 3 (7), 671674.

(27) Javier, A. E.; Varshney, S. R.; McCullough, R. D. Macromolecules 2010, 43, 3233-3237.

(28) Verduzco, R.; Botiz, I.; Pickel, D. L.; Kilbey, S. M.; Hong, K.; Dimasi, E.; Darling, S. B. Macromolecules 2011, 44 (3), 530-539.

(29) Bryan, Z. J.; Smith, M. L.; McNeil, A. J. Macromol. Rapid Commun. 2012, 33, 842-847.

(30) Wu, S.; Bu, L.; Huang, L.; Yu, X.; Han, Y.; Geng, Y.; Wang, F. Polymer (Guildf). 2009, $50(26), 6245-6251$.

(31) Verswyvel, M.; Monnaie, F.; Koeckelberghs, G. Macromolecules 2011, 44 (24), 94899498.

(32) Miyakoshi, R.; Yokoyama, A.; Yokozawa, T. Chem. Lett. 2008, 37 (10), 1022-1023.

(33) Buga, K.; Pokrop, R.; Zagórska, M.; Demadrille, R.; Genoud, F. Synth. Met. 2005, 153, $137-140$.

(34) Burkhart, B.; Khlyabich, P. P.; Thompson, B. C. Macromolecules 2012, 45, 3740-3748. 
(35) Hollinger, J.; Sun, J.; Gao, D.; Karl, D.; Seferos, D. S. Macromol. Rapid Commun. 2013, $34(5), 437-441$.

(36) Elmalem, E.; Kiriy, A.; Huck, W. T. S. Macromolecules 2011, 44, 9057-9061.

(37) Ono, R. J.; Kang, S.; Bielawski, C. W. Macromolecules 2012, 45 (5), 2321-2326.

(38) Hardeman, T.; Koeckelberghs, G. Macromolecules 2014, 47, 8618-8624.

(39) Bronstein, H.; Hurhangee, M.; Fregoso, E. C.; Beatrup, D.; Soon, Y. W.; Huang, Z.; Hadipour, A.; Tuladhar, P. S.; Rossbauer, S.; Sohn, E.; Shoaee, S.; Dimitrov, S. D.; Frost, J. M.; Ashraf, R. S.; Kirchartz, T.; Watkins, S. E.; Song, K.; Anthopoulos, T.; Nelson, J.; Rand, B. P.; Durrant, J. R.; Mcculloch, I. Chem. Mater. 2013, 25, 4239-4249.

(40) Verswyvel, M.; Verstappen, P.; De Cremer, L.; Verbiest, T.; Koeckelberghs, G. J. Polym. Sci. Part A Polym. Chem. 2011, 49 (24), 5339-5349.

(41) Verswyvel, M.; Steverlynck, J.; Mohamed, S. H.; Trabelsi, M. Macromolecules 2014, 47, 4668-4675.

(42) Tu, G.; Li, H.; Forster, M.; Heiderhoff, R.; Balk, L. J.; Sigel, R.; Scherf, U. Small 2007, 3 (6), 1001-1006.

(43) Palermo, E. F.; Mcneil, A. J. Macromolecules 2012, 45, 5948-5955.

(44) Palermo, E. F.; van der Laan, H. L.; McNeil, A. J. Polym. Chem. 2013, 4 (17), 4606.

(45) Locke, J. R.; McNeil, A. J. Macromolecules 2010, 43 (21), 8709-8710.

(46) Van den Bergh, K.; Cosemans, I.; Verbiest, T.; Koeckelberghs, G. Macromolecules 2010, 43 (8), 3794-3800.

(47) Skeist, I. J. Am. Chem. Soc. 1946, 68 (9), 1781-1784.

(48) Mayo, F. R.; Walling, C. Chem Revs. 1950, 46, 191-287. 
For Table of Contents use only

Copolymerization of thiophene and fluorene using the $\mathrm{Pd}(\mathrm{RuPhos})-$ protocol

Tine Hardeman, Guy Koeckelberghs*

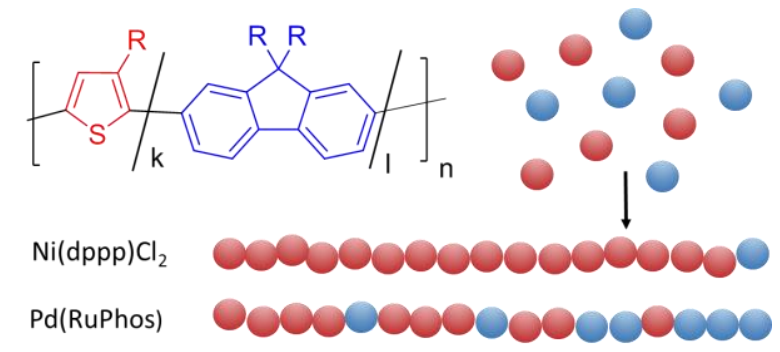

